\title{
Circulating hepassocin level in patients with stable angina is associated with fatty liver and renal function
}

\author{
I-Ting Tsai ${ }^{1,5}$, Wei-Chin Hung, 2,5 , Yung-Chuan $\mathrm{Lu}^{3,7}$, Cheng-Ching Wu2,6,11, Thung-Lip Lee ${ }^{2,7}$, Chin-Feng Hsuan ${ }^{2,5}$, \\ Teng-Hung Yu ${ }^{2,6}$, Ching-Ting Wei ${ }^{4,7,8,9}$, Fu-Mei Chung2 ${ }^{2}$, Yau-Jiunn Lee ${ }^{10}$, and Chao-Ping Wang ${ }^{2,7 凶}$ \\ 1. Department of Emergency, E-Da Hospital, Kaohsiung 82445 Taiwan. \\ 2. Division of Cardiology, Department of Internal Medicine, E-Da Hospital, Kaohsiung 82445 Taiwan. \\ 3. Division of Endocrinology and Metabolism, Department of Internal Medicine, E-Da Hospital, Kaohsiung 82445 Taiwan. \\ 4. Division of General Surgery, Department of Surgery, E-Da Hospital, Kaohsiung 82445 Taiwan. \\ School of Medicine, College of Medicine, I-Shou University, Kaohsiung, 82445 Taiwan. \\ The School of Chinese Medicine for Post Baccalaureate, College of Medicine, I-Shou University, Kaohsiung, 82445 Taiwan. \\ School of Medicine for International Students, College of Medicine, I-Shou University, Kaohsiung, 82445 Taiwan. \\ 8. Department of Biomedical Engineering, I-Shou University, Kaohsiung, 82445 Taiwan. \\ 9. Department of Electrical Engineering, I-Shou University, Kaohsiung, 82445 Taiwan. \\ 10. Lee's Endocrinologic Clinic, Pingtung 90000 Taiwan. \\ 11. Division of Cardiology, Department of Internal Medicine, E-Da Cancer Hospital, Kaohsiung 82445 Taiwan. \\ $\triangle$ Corresponding author: Dr. Chao-Ping Wang, E-Da Hospital, I-Shou University, No. 1, Yi-Da Rd., Jiau-Shu Village, Yan-Chao Township, Kaohsiung, 82445, Taiwan. Tel: \\ +886-7-615-1100 ext. 5018; E-mail: ed100232@edah.org.tw.
}

(C) The author(s). This is an open access article distributed under the terms of the Creative Commons Attribution License (https://creativecommons.org/licenses/by/4.0/). See http://ivyspring.com/terms for full terms and conditions.

Received: 2020.07.13; Accepted: 2020.10.10; Published: 2021.01.01

\begin{abstract}
Background: Chronic kidney disease (CKD) is a major risk factor for coronary artery disease and it is often associated with hepatic steatosis. Hepassocin (also known as hepatocyte-derived fibrinogen related protein or fibrinogen-like 1) is a novel hepatokine that causes hepatic steatosis and induces insulin resistance. However, the role of hepassocin in renal function status remains unclear. Our objective was to investigate the association of plasma hepassocin level with fatty liver and renal function status in patients with stable angina.

Methods: Plasma hepassocin levels were determined by enzyme-linked immunosorbent assays in 395 consecutive patients with stable angina. Renal function was defined as an estimated glomerular filtration rate (eGFR). Fatty liver was defined by ultrasonography and fibrosis-4 (FIB-4) index.

Results: With increasing hepassocin tertiles, patients had higher prevalence of fatty live, an increased waist-to-hip ratio, and neutrophil count, monocyte count, and FIB-4 index, higher levels of uric acid, blood urine nitrogen and higher sensitivity C-reactive protein. They also had incrementally lower eGFR, serum hemoglobin and albumin levels. In multiple linear stepwise regression analysis, only eGFR was significantly independent negatively associated with plasma hepassocin levels.

Conclusion: Our results indicate that circulating hepassocin in patients with stable angina is associated with fatty liver and renal function, which suggests that increased plasma hepassocin may be involved in the pathogenesis of fatty liver and CKD.
\end{abstract}

Key words: Hepassocin; fatty liver; renal function; stable angina

\section{Introduction}

Chronic kidney disease (CKD) is an independent risk factor for coronary artery disease [1]. There is a graded-independent association between reduced estimated glomerular filtration rate (eGFR) and the risk of death, cardiovascular events, and hospitalization [2]. CKD has been a global public health issue for the past few decades and it affects more than $10 \%$ of the worldwide population. The health burden of CKD is not restricted to the need for renal replacement therapy for end-stage renal disease (ESRD), but also its other serious outcomes, such as cardiovascular events and death, which are strongly influenced by kidney involvement [3,4]. Previous study reported that in 2010, death caused by CKD almost doubled compared with 1990 and it was ranked as the 18th highest risk factor for mortality [5]. Taiwan has the highest incidence of ESRD, which requires renal replacement therapy, and CKD 
contributes to this burden. CKD patients have a chronic, low grade, inflammatory condition that is mediated by inflammatory cytokines [6]. In the liver, hepatic inflammation triggers a cascade of hepatocyte injuries, ranging from simple hepatic steatosis to nonalcoholic steatohepatitis, cirrhosis and even hepatocellular carcinoma $[7,8]$. In addition, subclinical chronic inflammation seems to be an independent risk factor for the development of stable angina in CKD patients [9]. Although it is well known that CKD, nonalcoholic fatty liver disease (NAFLD) and cardiovascular disease are strongly connected, the factors linking these diseases are not yet fully understood.

Hepassocin, also known as hepatocyte-derived fibrinogen related protein or fibrinogen-like 1, is a specific mitogenic factor for hepatocytes. It has also been identified as a novel hepatokine, which increases hepatic lipogenesis that causes hepatic steatosis [10]. In addition, hepassocin was demonstrated to induce insulin resistance (IR) in both the liver [11] and skeletal muscles [12]. Although a previous study indicated that hepassocin is also expressed by adipose tissues, the association between hepassocin and renal function status remains obscure [13].

In view of the fact that hepassocin causes hepatic steatosis and induces IR, and because CKD has a clear association with both hepatic steatosis and type 2 diabetes [14,15], the authors postulated that hepassocin might be the link between these diseases. The aim of the present study was to clarify the clinical significance of circulating hepassocin levels in the context of fatty liver and renal function status. Circulating hepassocin levels were determined in patients with stable angina, and then the association between those levels and the fatty liver and renal function status were evaluated.

\section{Methods}

\section{Study participants}

A total of 414 consecutive patients with stable angina who were admitted to the cardiovascular ward of E-Da Hospital between June 2007 and June 2017 were prospectively enrolled in the study. The eGFRs were calculated using the CKD-EPI two-concentration race equation [16], and their renal function status was confirmed by follow-up eGFR measurements at 3 months after hospital discharge. Patients with a history of concomitant inflammatory disease (including malignancy, liver disease, sepsis, infection and collagen disease), steroid use or surgery within 1 month prior to admission, as determined by interviews, physical examinations, biochemical laboratory analysis and urinalysis, were excluded from the study. Furthermore, patients who were unable or unwilling to give informed consent were also excluded. After the exclusion of 19 patients, a total of 395 patients with stable angina were included in the present study. In addition, in the present study, no patients have undergone dialysis. Hypertension was defined as a resting systolic blood pressure of $\geq 140 \mathrm{mmHg}$, a diastolic blood pressure of $\geq 90 \mathrm{mmHg}$ or both, and those with a history of hypertension and the use of anti-hypertensive drugs. Diabetic patients were defined as those who were currently being treated for diabetes, those with a fasting plasma glucose level $\geq 126 \mathrm{mg} / \mathrm{dl}$ on 3 separate days, or a casual glucose level $\geq 200 \mathrm{mg} / \mathrm{dl}$. Hyperlipidemia was defined as a total cholesterol level of $>200 \mathrm{mg} / \mathrm{dl}$, and/or a low-density lipoprotein cholesterol (LDL-C) level of >130 mg/dl, and/or a high-density lipoprotein cholesterol (HDL-C) level of $<40 \mathrm{mg} / \mathrm{dl}$, and/or a triglycerides level of $>180 \mathrm{mg} / \mathrm{dl}$, or those undergoing treatment for lipid disorders. The study protocol was approved by the Human Research Ethics Committee at E-Da Hospital and all patients provided written informed consent before enrollment.

In the present study, all of the study patients were of Han Chinese origin and lived in the same region. All patients underwent complete routine biochemical analyses of their blood and urine, as well as physical examinations. Anthropometric parameters were taken, including their waist-to-hip ratio and their body mass index (BMI). Waist and hip circumferences were measured at the end of a normal respiration and each measurement was repeated twice. The measurements were recorded to the nearest $0.1 \mathrm{~cm}$ and the waist was measured at the narrowest point between the lower border of the ribs and the right iliac crest. Hip circumferences were measured at the widest point. Seated blood pressure was also measured by a trained nurse with a digital automatic blood pressure monitor (Omron model HEM-907, Omron, Japan) after the patients had rested for 5 minutes. Plasma biochemical parameters were measured after overnight fasting. Plasma total cholesterol, LDL-C, HDL-C, triglycerides, creatinine, glucose, and uric acid were measured using standard commercial methods as described in our previous reports $[17,18]$. Patients who had smoked within one year of the examination were defined as current smokers and those who had stopped smoking for more than one year before the examination were defined as nonsmokers.

\section{Plasma hs-CRP and hepassocin measurements}

All patient blood samples were drawn after overnight fasting, and all plasma samples were kept at $-80{ }^{\circ} \mathrm{C}$ prior to subsequent assays. Plasma 
hepassocin concentration was measured using a commercial enzyme-linked immunosorbent assay (ELISA) kit (intra-assay CV $<10 \%$, inter-assay CV $<12 \%$; Cloud-Clone Corp., Katy, USA) and the ELISA was performed according to the manufacturer's instructions. According to the manufacturer, the hepassocin ELISA has excellent specificity for the detection of human hepassocin, and no significant interference with analogues or cross-reactivity was observed. In addition, plasma high sensitivity C-reactive protein (hs-CRP) was determined using a Beckman Coulter IMMAGE Immunochemistry System (Brea, CA, USA). Samples were measured in duplicate in a single experiment and the detection limit of the hs-CRP assay system was $0.2 \mathrm{mg} / \mathrm{L}$.

\section{Liver ultrasonography and fibrosis-4 (FIB-4) index calculation}

All participants underwent hepatic ultrasonography, which was performed by two experienced physicians who were blinded to the study aims. Hepatic steatosis was diagnosed according to the following sonographic features: evidence of diffuse hyperechogenicity in the liver relative to the kidneys, poor visualization of intrahepatic structures, and ultrasound beam attenuation. The FIB-4 index was calculated as follows: FIB-4 = age (years) $\times$ AST (IU/L) / platelet count $\left(10^{9} / \mathrm{L}\right) / \sqrt{ }$ ALT $(\mathrm{IU} / \mathrm{L})$, as previously reported by Vallet-Pichard et al. [19].

\section{Statistical analysis}

Data normality was analyzed using the Kolmogorov-Smirnov test. Continuous, normally distributed variables are described as the mean \pm standard deviation. Non-normally distributed variables are described as the median (interquartile range). Statistical differences between normally distributed variables were compared using one-way analysis of variance (ANOVA) followed by Tukey pairwise comparison. Categorical variables are presented as frequencies and percentages, and intergroup comparisons were tested using the chi-square test. Logarithmically transformed values of plasma hepassocin, hs-CRP, blood urine nitrogen (BUN), creatinine, and triglyceride were used in the statistical analyses since their distributions were skewed. Associations between hepassocin and other parameters were assessed using simple and multiple linear stepwise regression analyses. Results were considered statistically significant if their $p$ value was $<0.05$. All analyses were performed using SAS statistical software, version 8.2 (SAS Institute Inc., Cary, NC, USA).

\section{Results}

\section{Main characteristics according to tertiles of hepassocin}

Table 1 shows the clinical characteristics of the 395 patients with stable angina (males, 69.6\%; females, $30.4 \%$ ) stratified by hepassocin. The mean hepassocin level was $1212.4 \mathrm{ng} / \mathrm{mL}$ and the median plasma hepassocin level was $903.0 \mathrm{ng} / \mathrm{mL}$ (interquartile range, 431.6-1413.9 $\mathrm{ng} / \mathrm{mL}$ ). The patients were divided according to the tertiles of hepassocin as follows: low hepassocin ( $\leq 570 \mathrm{ng} / \mathrm{mL}), \mathrm{n}=130$; medium hepassocin (571-1230 ng/mL), $\mathrm{n}=132$; and high hepassocin $(>1230 \mathrm{ng} / \mathrm{mL}), \mathrm{n}=133$. The patients in the high hepassocin group were associated with higher prevalence of fatty liver and lower prevalence of single-vessel disease. Furthermore, the high hepassocin group had a higher waist-to-hip ratio compared with the low hepassocin group. There were no significant differences in sex, age, hypertension, diabetes mellitus, hyperlipidemia, current smoker, BMI, SBP, DBP, left ventricular ejection fraction, medications, two-vessel disease, and three-vessel disease among the three groups.

\section{Biochemical characteristics according to tertiles of hepassocin}

The data presented in Table 2 shows that as the hepassocin tertile increased, there were significant decreases in eGFR, hemoglobin and albumin concentrations, and significant increases in uric acid, BUN, and hs-CRP concentrations, as well as neutrophil and monocyte count and FIB-4 index. There were no significant differences in fasting glucose, HbA1C, triglyceride, HDL-cholesterol, LDL-cholesterol, creatinine, total WBC count, and lymphocyte count among the three groups.

\section{Association between plasma hepassocin levels and clinical laboratory data}

The results of the association analysis for circulating hepassocin and the relevant parameters are shown in Table 3. Plasma hepassocin was significantly positively related to uric acid $(\beta=0.137$, $p=0.010)$, BUN $(\beta=0.139, p=0.006)$, creatinine $(\beta=$ $0.103, p=0.040)$, total WBC count $(\beta=0.111, p=0.028)$, neutrophil count $(\beta=0.106, p=0.036)$, hs-CRP $(\beta=$ $0.108, p=0.041)$, and FIB-4 index $(\beta=0.122, p=0.012)$, and significantly negatively related to eGFR $(\beta=$ $-0.183, p<0.0001$, Figure 1) in the simple linear regression analysis with pooled data, whereas in the multiple linear stepwise regression analysis, only the plasma hepassocin level remained negatively associated with eGFR $(\beta=-0.228, p<0.0001)$. 


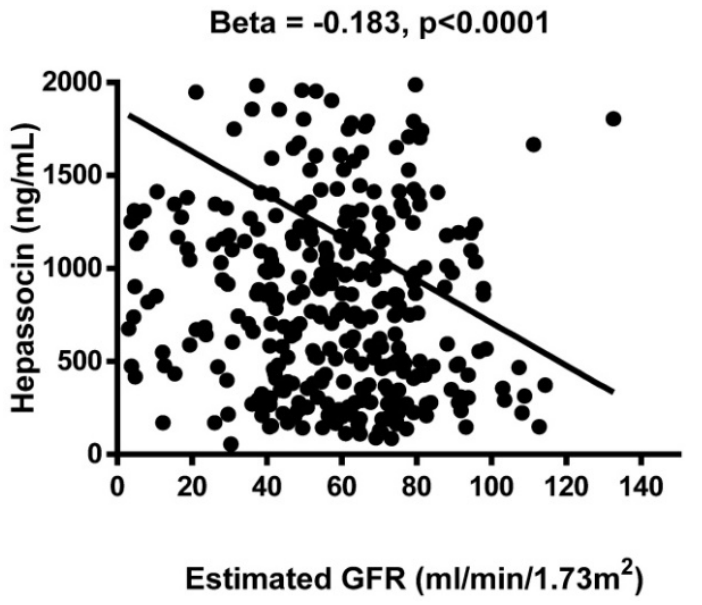

Figure 1. Association between plasma hepassocin concentration and estimated glomerular filtration rate (eGFR), plasma hepassocin concentration had a significant and negatively association with eGFR.

Table 1. Main characteristics according to tertiles of hepassocin

\begin{tabular}{|c|c|c|c|c|}
\hline Parameter & $\begin{array}{l}\text { Low } \\
\text { hepassocin } \\
\leq 570 \mathrm{ng} / \mathrm{mL}\end{array}$ & $\begin{array}{l}\text { Medium } \\
\text { hepassocin } \\
=571-1230 \mathrm{ng} / \mathrm{mL}\end{array}$ & $\begin{array}{l}\text { High } \\
\text { hepassocin } \\
>1230 \mathrm{ng} / \mathrm{mL}\end{array}$ & $\begin{array}{l}p \text { - } \\
\text { value }\end{array}$ \\
\hline No. & 130 & 132 & 133 & \\
\hline Sex (male/female) & $81 / 49$ & $99 / 33$ & $95 / 38$ & 0.071 \\
\hline Age (years) & $69.0 \pm 11.5$ & $69.8 \pm 10.4$ & $72.2 \pm 11.5$ & 0.054 \\
\hline Hypertension (n, \%) & $90(69.2)$ & $106(80.3)$ & $103(77.4)$ & 0.096 \\
\hline Diabetes mellitus (n, \%) & $50(38.5)$ & $60(45.5)$ & $62(46.6)$ & 0.355 \\
\hline Hyperlipidemia (n, \%) & $74(56.9)$ & $89(67.4)$ & $78(58.7)$ & 0.173 \\
\hline Current smoker (n, \%) & $38(29.2)$ & $52(39.4)$ & $50(37.6)$ & 0.186 \\
\hline Body mass index $\left(\mathrm{kg} / \mathrm{m}^{2}\right)$ & $25.7 \pm 4.7$ & $25.6 \pm 4.0$ & $24.9 \pm 4.1$ & 0.267 \\
\hline Waist-to-hip ratio & $0.92 \pm 0.08$ & $0.94 \pm 0.07$ & $0.94 \pm 0.08$ & 0.038 \\
\hline Systolic BP (mmHg) & $132 \pm 20$ & $133 \pm 23$ & $133 \pm 23$ & 0.877 \\
\hline Diastolic BP (mmHg) & $76 \pm 12$ & $77 \pm 11$ & $75 \pm 13$ & 0.565 \\
\hline $\operatorname{LVEF}(\%)$ & $60.7 \pm 14.3$ & $59.9 \pm 12.6$ & $60.0 \pm 15.3$ & 0.938 \\
\hline \multicolumn{5}{|l|}{ Medications (n, \%) } \\
\hline ACEIs/ARBs & $9(6.9)$ & $13(9.9)$ & $11(8.3)$ & 0.693 \\
\hline CCBs & $38(29.2)$ & $53(40.2)$ & $51(38.4)$ & 0.143 \\
\hline Diuretics & $35(26.9)$ & $40(30.3)$ & $39(29.3)$ & 0.825 \\
\hline Thiazolidinediones & $6(4.6)$ & $5(3.8)$ & $6(4.5)$ & 0.937 \\
\hline Insulin & $3(2.3)$ & $4(3.0)$ & $1(0.8)$ & 0.404 \\
\hline Statins use & $68(52.3)$ & $72(54.6)$ & $57(42.9)$ & 0.142 \\
\hline \multicolumn{5}{|c|}{ Number of diseased vessels (\%) } \\
\hline Single-vessel disease & $33(25.4)$ & $19(14.4)$ & $18(13.5)$ & 0.020 \\
\hline Two-vessel disease & $33(25.4)$ & $24(18.2)$ & $32(24.1)$ & 0.330 \\
\hline Three-vessel disease & $45(34.6)$ & $61(46.2)$ & $53(39.9)$ & 0.159 \\
\hline \multicolumn{5}{|l|}{ Fatty liver $(\%)$} \\
\hline Yes & $41(31.1)$ & $46(35.4)$ & $63(47.4)$ & 0.018 \\
\hline No & $91(68.9)$ & $84(64.6)$ & $70(52.6)$ & \\
\hline
\end{tabular}

Data are expressed as the mean $\pm \mathrm{SD}$, or number (percentage). $\mathrm{BP}$, blood pressure; $\mathrm{LVEF}$, left ventricular ejection fraction; ACEI, angiotensin- converting enzyme inhibitor; $\mathrm{ARB}$, angiotensin receptor blocker; $\mathrm{CCB}$, calcium channel blocker. Fatty liver was defined by ultrasonography and fibrosis- 4 index.

\section{Discussion}

The present study demonstrated for the first time that plasma hepassocin levels associated with renal function status in patients with stable angina. Simple linear regression analysis revealed that hepassocin was positively associated with uric acid, BUN, creatinine, total WBC count, neutrophil count, hs-CRP, and FIB-4 index, and significantly negatively related to eGFR. Furthermore, in multiple linear stepwise regression analysis, only the plasma hepassocin level remained negatively associated with eGFR. With increasing hepassocin levels, the patients had higher rates of fatty liver, an increased waist-tohip ratio, neutrophil count and monocyte count, and higher levels of uric acid and BUN as well as hs-CRP and FIB-4 index. With increasing hepassocin levels, patients also had incrementally lower eGFR, serum hemoglobin and albumin levels.

In the current study, the associations between plasma hepassocin levels and other relevant risk factors for renal function were evaluated. Hepassocin is a hepatokine [20] that plays an important role in the regulation of hepatocyte proliferation [21], and its expression is increased during liver regeneration [22]. Previous study showed that in the regenerating liver may induce an acute-phase response and increased acute-phase cytokines [23]. These cytokines may contribute to CKD. In addition, a previous study provided evidence that hepassocin plays an important role in NAFLD and induces hepatic lipid accumulation through an extracellular signalregulated kinase 1/2 (ERK1/2)-dependent signaling pathway [24]. Lu et al. reported that serum hepassocin concentrations are gradually increased in diabetic patients with NAFLD, and suggested that increased hepassocin levels might have clinical implications and could play a role as a biomarker for diabetes and NAFLD [25]. Our recent study showed that a bidirectional relationship existed between CKD and NAFLD in patients with type 2 diabetes mellitus [14]. Consistent with these findings, it was found in the present study that patients in the high hepassocin group were associated with increased rates of fatty liver and decreased eGFR.

Hepassocin is also expressed by brown adipose tissues, and signals generated following liver injury can also enhance hepassocin expression by brown adipose tissues, which suggests that there is cross talk between the injured liver and adipose tissues [26]. In addition, it is known that interleukin-6 (IL-6) increases hepassocin expression [27], indicating that elevated hepassocin might also be involved in obesity-induced IR. Furthermore, Cheng et al. found that activation of signal transducer and activator of transcription- 3 by IL- 6 were mediated by hepassocin expression [28]. In the current study, it was revealed that the high hepassocin group had a higher waist-to-hip ratio compared with the low hepassocin group, and with increasing hepassocin tertiles, there were significant increases in hs-CRP concentrations as well as neutrophil and monocyte counts. The results of the present study support the idea [29-31] that hepassocin may act through the IR and inflammation response to play an important role in the impact of renal function status in patients with stable angina. 
Table 2. Biochemical characteristics according to tertiles of hepassocin

\begin{tabular}{|c|c|c|c|c|}
\hline Parameter & Low hepassocin $\leq 570 \mathrm{ng} / \mathrm{mL}$ & Medium hepassocin $=571-1230 \mathrm{ng} / \mathrm{mL}$ & High hepassocin $>1230 \mathrm{ng} / \mathrm{mL}$ & $p$-value \\
\hline No. & 130 & 132 & 133 & \\
\hline Fasting glucose $(\mathrm{mg} / \mathrm{dl})$ & $133.5 \pm 68.4$ & $136.4 \pm 61.8$ & $137.1 \pm 60.3$ & 0.888 \\
\hline $\operatorname{HbA} 1 \mathrm{C}(\%)$ & $6.7 \pm 1.4$ & $6.9 \pm 1.4$ & $7.0 \pm 1.6$ & 0.233 \\
\hline T-cholesterol (mg/dl) & $169.1 \pm 37.8$ & $180.5 \pm 46.5$ & $167.6 \pm 42.6$ & 0.028 \\
\hline Triglyceride (mg/dl) & $115.0(83.0-173.5)$ & $118.0(92.0-180.0)$ & $109.0(78.3-164.3)$ & 0.261 \\
\hline HDL-cholesterol (mg/dl) & $40.6 \pm 12.3$ & $39.4 \pm 11.0$ & $39.2 \pm 11.7$ & 0.558 \\
\hline LDL-cholesterol (mg/dl) & $100.0 \pm 35.6$ & $106.7 \pm 36.0$ & $98.5 \pm 35.3$ & 0.143 \\
\hline Uric acid $(\mathrm{mg} / \mathrm{dl})$ & $6.2 \pm 2.0$ & $6.8 \pm 1.9$ & $7.1 \pm 2.7$ & 0.006 \\
\hline BUN (mg/dl) & $17.3(13.9-22.2)$ & $19.6(15.9-26.6)$ & $20.0(14.1-29.4)$ & 0.027 \\
\hline Creatinine $(\mathrm{mg} / \mathrm{dl})$ & $1.2(1.0-1.4)$ & $1.3(1.1-1.7)$ & $1.3(1.1-1.8)$ & 0.053 \\
\hline eGFR $\left(\mathrm{ml} / \mathrm{min} / 1.73 \mathrm{~m}^{2}\right)$ & $63.2 \pm 22.6$ & $53.8 \pm 22.0$ & $51.9 \pm 24.3$ & 0.0001 \\
\hline Hemoglobin $(\mathrm{g} / \mathrm{dL})$ & $13.1 \pm 1.9$ & $13.1 \pm 1.9$ & $12.6 \pm 2.1$ & 0.049 \\
\hline Albumin (g/dL) & $4.0 \pm 0.4$ & $3.9 \pm 0.4$ & $3.8 \pm 0.4$ & 0.036 \\
\hline Total WBC count $\left(10^{9} / \mathrm{L}\right)$ & $7.059 \pm 2.399$ & $7.402 \pm 2.469$ & $7.759 \pm 3.231$ & 0.065 \\
\hline Neutrophil count $\left(10^{9} / \mathrm{L}\right)$ & $4441 \pm 2147$ & $4569 \pm 2162$ & $5150 \pm 2929$ & 0.044 \\
\hline Monocyte count $\left(10^{9} / \mathrm{L}\right)$ & $397 \pm 189$ & $427 \pm 182$ & $574 \pm 303$ & 0.041 \\
\hline Lymphocyte count $\left(10^{9} / \mathrm{L}\right)$ & $2004 \pm 893$ & $2069 \pm 970$ & $1894 \pm 715$ & 0.253 \\
\hline hs-CRP (mg/L) & $1.8(0.7-5.8)$ & $2.8(1.0-8.9)$ & $3.3(1.2-10.1)$ & 0.008 \\
\hline Fibrosis-4 index & $1.1(0.9-1.4)$ & $1.8(1.4-2.4)$ & $3.2(2.3-4.5)$ & 0.0001 \\
\hline
\end{tabular}

Table 3. Linear regression analysis of variables associated with plasma hepassocin levels

\begin{tabular}{|c|c|c|c|c|}
\hline \multirow[t]{2}{*}{ Variable } & \multicolumn{2}{|l|}{ Simple } & \multicolumn{2}{|l|}{ Multiple } \\
\hline & $\beta$ coefficient & $p$-value & $\beta$ coefficient & $p$-value \\
\hline Body mass index & -0.032 & 0.520 & - & - \\
\hline Systolic BP & 0.057 & 0.264 & - & - \\
\hline Diastolic BP & -0.005 & 0.926 & - & - \\
\hline Fasting sugar & 0.022 & 0.666 & - & - \\
\hline $\mathrm{HbA1C}$ & 0.042 & 0.425 & - & - \\
\hline T-cholesterol & -0.024 & 0.639 & - & - \\
\hline Triglyceride & 0.099 & 0.050 & - & - \\
\hline HDL-cholesterol & -0.033 & 0.516 & - & - \\
\hline LDL-cholesterol & -0.053 & 0.293 & - & - \\
\hline Uric acid & 0.137 & 0.010 & - & - \\
\hline Blood urea nitrogen & 0.139 & 0.006 & - & - \\
\hline Creatinine & 0.103 & 0.040 & - & - \\
\hline eGFR & -0.183 & $<0.0001$ & -0.228 & $<0.0001$ \\
\hline Hemoglobin & -0.092 & 0.067 & - & - \\
\hline Albumin & -0.022 & 0.675 & - & - \\
\hline Total WBC count & 0.111 & 0.028 & - & - \\
\hline Neutrophil count & 0.106 & 0.036 & - & - \\
\hline Monocyte count & 0.044 & 0.384 & - & - \\
\hline Lymphocyte count & -0.020 & 0.699 & - & - \\
\hline Hs-CRP & 0.108 & 0.041 & - & - \\
\hline Fibrosis-4 index & 0.122 & 0.012 & - & - \\
\hline
\end{tabular}

To the best of our knowledge, this is the first report to demonstrate that hepassocin levels are associated with renal function status. The biological mechanisms by which hepassocin is involved in the impact of renal function are not well understood. The current study found an inverse correlation between plasma hepassocin levels and eGFR, however, whether unchanged hepassocin levels reflect impaired hepassocin clearance by the kidneys or a compensatory mechanism aimed at counteracting increased cardiovascular risk factors, is not clear. Previous studies have reported that hepassocin regulates cell fate and has a protective effect on CCl4-induced liver injury [32]. In addition to hepassocin's role in the development of NAFLD, the hepassocin promoter is transcriptionally upregulated through an IL-6/IL-6R/STAT3 signaling pathway, which maintains metabolic homeostasis [33]. In addition, NAFLD is strongly associated with obesity, diabetes and IR, and is considered to be the hepatic manifestation of metabolic syndrome [34,35]. Hepassocin may therefore play a role in the regulation of insulin sensitivity or metabolic homeostasis. On the basis of these reports, patients with stable angina with elevated hepassocin levels had higher prevalence of fatty live and reduced renal function observed in the current study, could indicate that hepassocin may be associated with pathogenesis of fatty live and CKD.

There were several limitations to the current study. First, the study was cross-sectional in design, and it was not possible to clearly establish a causal link between plasma hepassocin levels and the development of CKD. Second, it is well known that the use of lipid-lowering agents and anti-diabetic medicines influences CKD. The association between patients receiving prescription medications among the tertiles of hepassocin was analyzed. There were no significant differences in angiotensin-converting enzyme inhibitors/angiotensin receptor blockers, calcium channel blocker, diuretics, thiazolidinediones, and statins treatments among the three groups. Hence, the possible influence of these medications on renal function cannot be ignored. 
Third, the current study included only patients with stable angina, and therefore the results may not be fully applicable to the general population. Fourth, the hepassocin molecular weight is $36 \mathrm{kDa}$ that is belong to low molecular weight protein. Low molecular weight proteins may possibly eliminated by the kidney. However, previous studies demonstrated that low molecular weight human serum proteins, peptides, and other small components have been associated with pathological conditions such as cancer [36], diabetes [37], and cardiovascular and infectious diseases [38], and kidney disease [39]. Hence, according to the results of the present study, hepassocin may play a role in the impact of renal function. But, further studies are needed to clarify the exact role of hepassocin in renal function status. Fifth, hepassocin expression is increased during liver regeneration and so hepassocin may be as an acute phase protein. Further work is required to confirm the other proteins including fibrinogen that could be of some help in clarifying the role of hepassocin as an acute phase protein and impact of renal function status. Finally, the analyses are based on single measurements of plasma hepassocin, which may not fully reflect the association over time. It would be interesting to measure serial changes in plasma hepassocin levels in CKD, obese or IR subjects to further clarify the role of hepassocin in the pathogenesis of CKD. Additional studies that include a larger multi-ethnic cohort are needed to investigate these associations.

\section{Conclusions}

In conclusion, our findings of elevated hepassocin levels were associated fatty liver and renal function status, indicate that hepassocin may contributed to the pathogenesis of fatty liver and CKD in patients with stable angina. However, further studies are needed to clarify the exact role of hepassocin.

\section{Acknowledgements}

The authors would like to thank the E-Da Hospital, Republic of China, Taiwan, for financially supporting this research under contracts EDAHI107002, EDAHP107052 and EDAHI108001. The authors appreciate the help of the staff and the members of the heart care teams for their assistance in taking various measurements and other organizational aspects of this study.

\section{Competing Interests}

The authors have declared that no competing interest exists.

\section{References}

1. Ix JH, Shlipak MG, Liu HH, Schiller NB, Whooley MA. Association between renal insufficiency and inducible ischemia in patients with coronary artery disease: the heart and soul study. J Am Soc Nephrol. 2003; 14: 3233.

2. Go AS, Chertow GM, Fan D, McCulloch CE, Hsu CY. Chronic kidney disease and the risks of death, cardiovascular events, and hospitalization. N Engl J Med. 2004; 351: 1296-305

3. Bello AK, Nwankwo E, El Nahas AM. Prevention of chronic kidney disease: a global challenge. Kidney Int Suppl. 2005; (98): S11-7.

4. Nugent RA, Fathima SF, Feigl AB, Chyung D. The burden of chronic kidney disease on developing nations: a 21st century challenge in global health. Nephron Clin Pract. 2011; 118: c269-77.

5. Lozano R, Naghavi M, Foreman K, Lim S, Shibuya K, Aboyans V, et al. Global and regional mortality from 235 causes of death for 20 age groups in 1990 and 2010: a systematic analysis for the Global Burden of Disease Study 2010. Lancet. 2012; 380: 2095-128.

6. Panichi V, Migliori M, De Pietro S, Taccola D, Bianchi AM, Norpoth M, et al. C reactive protein in patients with chronic renal diseases. Ren Fail. 2001; 23: 551-62.

7. Assunção SNF, Sorte NCB, Alves CD, Mendes PSA, Alves CRB, Silva LR. Nonalcoholic fatty liver disease (NAFLD) pathophysiology in obese children and adolescents: Update. Nutr Hosp. 2017; 34: 727-30.

8. Anstee QM, Daly AK, Day CP. Genetic modifiers of nonalcoholic fatty liver disease progression. Biochim Biophys Acta. 2011; 1812: 1557-66.

9. Nusair MB, Rajpurohit N, Alpert MA. Chronic Inflammation and Coronary Atherosclerosis in Patients with End-Stage Renal Disease. Cardiorenal Med. 2012; 2: 117-24.

10. Wu HT, Lu FH, Ou HY, Su YC, Hung HC, Wu JS, et al. The role of hepassocin in the development of nonalcoholic fatty liver disease. J Hepatol. 2013; 59: 1065-72.

11. Wu HT, Ou HY, Hung HC, Su YC, Lu FH, Wu JS, et al. A novel hepatokine, HFREP1, plays a crucial role in the development of insulin resistance and type 2 diabetes. Diabetologia. 2016; 59: 1732-42.

12. Jung TW, Chung YH, Kim HC, El-Aty AMA, Jeong JH. Hyperlipidemiainduced hepassocin in the liver contributes to insulin resistance in skeletal muscle. Mol Cell Endocrinol. 2018; 470: 26-33.

13. Axelsson J, Heimbürger $O$, Stenvinkel P. Adipose tissue and inflammation in chronic kidney disease. Contrib Nephrol. 2006; 151: 165-74.

14. Lee YJ, Wang CP, Hung WC, Tang WH, Chang YH, Hu DW, et al. Common and unique factors, and the bidirectional relationship between chronic kidney disease and nonalcoholic fatty liver in type 2 diabetes patients. Diabetes Metab Syndr Obes. 2020; 13: 1203-14

15. Jepson C, Hsu JY, Fischer MJ, Kusek JW, Lash JP, Ricardo AC, et al.; Chronic Renal Insufficiency Cohort (CRIC) Study Investigators. Incident Type 2 Diabetes among Individuals with CKD: Findings from the Chronic Renal Insufficiency Cohort (CRIC) Study. Am J Kidney Dis. 2019; 73: 72-81.

16. Kong X, Ma Y, Chen J, Luo Q, Yu X, Li Y, et al.; Chinese eGFR Investigation Collaboration. Evaluation of the chronic kidney disease epidemiology collaboration equation for estimating glomerular filtration rate in the Chinese population. Nephrol Dial Transplant. 2013; 28: 641-51.

17. Tsai IT, Wang CP, Yu TH, Lu YC, Lin CW, Lu LF, et al. Circulating visfatin level is associated with hepatocellular carcinoma in chronic hepatitis B or C virus infection. Cytokine. 2017; 90: 54-9.

18. Lu LF, Tang WH, Hsu CC, Tsai IT, Hung WC, Yu TH, et al. Associations among chronic kidney disease, high total $p$-cresylsulfate and left ventricular systolic dysfunction. Clin Chim Acta. 2016; 457: 63-8.

19. Vallet-Pichard A, Mallet V, Nalpas B, Verkarre V, Nalpas A, Dhalluin-Venier $\mathrm{V}$, et al. FIB-4: an inexpensive and accurate marker of fibrosis in HCV infection. Comparison with liver biopsy and fibrotest. Hepatology. 2007; 46: 32-6.

20. Hara H, Yoshimura H, Uchida S, Toyoda Y, Aoki M, Sakai Y, et al. Molecular cloning and functional expression analysis of a cDNA for human hepassocin, a liver-specific protein with hepatocyte mitogenic activity. Biochim Biophys Acta. 2001; 1520: 45-53

21. Cao MM, Xu WX, Li CY, Cao CZ, Wang ZD, Yao JW, et al. Hepassocin regulates cell proliferation of the human hepatic cells 102 and hepatocarcinoma cells through different mechanisms. J Cell Biochem. 2011; 112: 2882-90.

22. Hara H, Uchida S, Yoshimura H, Aoki M, Toyoda Y, Sakai Y, et al. Isolation and characterization of a novel liver-specific gene, hepassocin, upregulated during liver regeneration. Biochim Biophys Acta. 2000; 1492: 31-44.

23. Batusic DS, von Bargen A, Blaschke S, Dudas J, Ramadori G. Different physiology of interferon- $\alpha /-\gamma$ in models of liver regeneration in the rat. Histochem Cell Biol. 2011; 136: 131-44.

24. Wu HT, Lu FH, Ou HY, Su YC, Hung HC, Wu JS, et al. The role of hepassocin in the development of non-alcoholic fatty liver disease. J Hepatol. 2013; 59: 1065-72.

25. Lu FH, Ou HY, Wu HT, Huerta R, Carratala A, Nuñez J, et al. Serum hepassocin concentrations in diabetic patients with or without nonalcoholic fatty liver disease. Diabetes Management. 2014; 4: 255-61.

26. Demchev V, Malana G, Vangala D, Stoll J, Desai A, Kang HW, et al. Targeted deletion of fibrinogen like protein 1 reveals a novel role in energy substrate utilization. PLoS One. 2013; 8: e58084.

27. Liu Z, Ukomadu C. Fibrinogen-like protein 1, a hepatocyte derived protein is an acute phase reactant. Biochem Biophys Res Commun. 2008; 365: 729-34. 
28. Cheng KP, Ou HY, Hung HC, $\mathrm{Li} \mathrm{CH}$, Fan KC, Wu JS, et al. Unsaturated fatty acids increase the expression of hepassocin through a signal transducer and activator of transcription 3-dependent pathway in HepG2 cells. Lipids. 2018; 53: 863-9.

29. Spoto B, Pisano A, Zoccali C. Insulin resistance in chronic kidney disease: a systematic review. Am J Physiol Renal Physiol. 2016; 311: F1087-108.

30. Akchurin OM, Kaskel F. Update on inflammation in chronic kidney disease. Blood Purif. 2015; 39: 84-92.

31. Landray MJ, Wheeler DC, Lip GY, Newman DJ, Blann AD, McGlynn FJ, et al. Inflammation, endothelial dysfunction, and platelet activation in patients with chronic kidney disease: the chronic renal impairment in Birmingham (CRIB) study. Am J Kidney Dis. 2004; 43: 244-53.

32. Gao M, Zhan YQ, Yu M, Ge CH, Li CY, Zhang JH, et al. Hepassocin activates the EGFR/ERK cascade and induces proliferation of L02 cells through the Src-dependent pathway. Cell Signal. 2014; 26: 2161-6.

33. Yu HT, Yu M, Li CY, Zhan YQ, Xu WX, Li YH, et al. Specific expression and regulation of hepassocin in the liver and down-regulation of the correlation of HNF1alpha with decreased levels of hepassocin in human hepatocellular carcinoma. J Biol Chem. 2009; 284: 13335-47.

34. Lewis JR, Mohanty SR. Non-alcoholic fatty liver disease: a review and update. Dig Dis Sci. 2010; 55: 560-78.

35. Lazo M, Clark JM. The epidemiology of non-alcoholic fatty liver disease: a global perspective. Semin Liver Dis. 2008; 28: 339-50.

36. Petricoin EF, Ardekani AM, Hitt BA, Levine PJ, Fusaro VA, Steinberg SM, et al. Use of proteomic patterns in serum to identify ovarian cancer. Lancet. 2002; 359: $572-7$.

37. Basso D, Valerio A, Seraglia R, Mazza S, Piva MG, Greco E, et al. Putative pancreatic cancer-associated diabetogenic factor: 2030 MW peptide. Pancreas. 2002; 24: 8-14

38. Rubin RB, Merchant M. A rapid protein profiling system that speeds study of cancer and other diseases. Am Clin Lab. 2000; 19: 28-9.

39. Clark WR, Gao D. Low-molecular weight proteins in end-stage renal disease: potential toxicity and dialytic removal mechanisms. J Am Soc Nephrol. 2002; 13: $541-7$ 